\title{
Scanning Electron Microscopy Cathodoluminescence Studies of Piezoelectric Fields in an InGaN Multiple Quantum Well Light-Emitting Diode
}

\author{
K.L. Bunker, R. Garcia, P.E. Russell \\ Materials Science and Engineering Department and Analytical Instrumentation Facility, \\ North Carolina State University, 2410 Campus Shore Drive, 318 EGRC, Raleigh, NC 27695
}

This work involves the development and application of a Scanning Electron Microscopy (SEM)based Cathodoluminescence (CL) system for the investigation of piezoelectric fields in an indium gallium nitride (InGaN)-based optoelectronic device. SEM-based CL experiments in a Hitachi S$3200 \mathrm{~N}$ were used to study the influence of piezoelectric fields on the luminescence properties of a commercial Cree, Inc. InGaN-based MQW X-Bright ${ }^{\circledR}$ green Light Emitting Diode [1]. The existence and direction of the piezoelectric field in the InGaN-based device were investigated using SEM-CL peak voltage dependence and carrier generation density (i.e. beam current) dependence studies. Supporting evidence for the existence of a piezoelectric field in the device was determined from forward bias electroluminescence experiments.

A compact SEM-CL spectroscopy system with both polychromatic spectroscopic and panchromatic imaging capabilities was designed and implemented for these experiments (Fig. 1). The CL polychromatic spectroscopy setup consisted of a USB2000 Ocean Optics miniature spectrometer coupled via a fiber optic light collection system (Fig. 1 and 2). The spectrometer is a 2048-element linear CCD array and was configured with a $50 \mu \mathrm{m}$ slit, a 600 lines $/ \mathrm{mm}$ grating, a spectral window between $350 \mathrm{~nm}-1000 \mathrm{~nm}$, and $2 \mathrm{~nm}$ optical resolution. A 1000 micron fused silica fiber optic terminated with a SMA 905 connector was connected to the spectrometer via a vacuum feedthrough. A $5.1 \mathrm{~mm}^{2} \mathrm{Si}$ photodetector on a TO-5 header mount was used to collect panchromatic CL images (Fig. 1 and 2). The custom sample holder was designed to allow simultaneous placement and positioning of the fiber optic and Si photodetector as well as provide electrical contact to the sample.

The CL emission peak showed a blueshift with increasing reverse bias due to the cancellation of the piezoelectric field (Fig. 3). A full compensation of the piezoelectric field was observed followed by a redshift with a further increase of reverse bias, indicating that flat band conditions had been reached. We determined the piezoelectric fields point in the [000-1] direction and estimated the magnitude to be approximately $1.0 \pm 0.2 \mathrm{MV} / \mathrm{cm}$ by calculating the depletion region thickness from the donor and acceptor concentrations, the built-in potential, and the applied voltage. A blueshift of the CL emission peak was also observed as the carrier generation density increased or the beam current increased (and hence the total carrier injection level) (Fig. 3). In the EL experiment, a $10 \mathrm{~nm}$ blueshift was observed as the forward bias was increased from 2.1 volts to 5.0 volts. The blueshift phenomenon observed in the SEM-CL carrier generation and EL experiments is due to the screening or weakening of the piezoelectric field by the injected or beam generated carriers in the quantum well.

\section{References}

[1] K.L. Bunker et al., 'Scanning electron microscopy cathodoluminescence studies of piezoelectric fields in an InGaN/GaN quantum well light-emitting diode', Applied Physics Letters, (in press, February 21, 2005). 

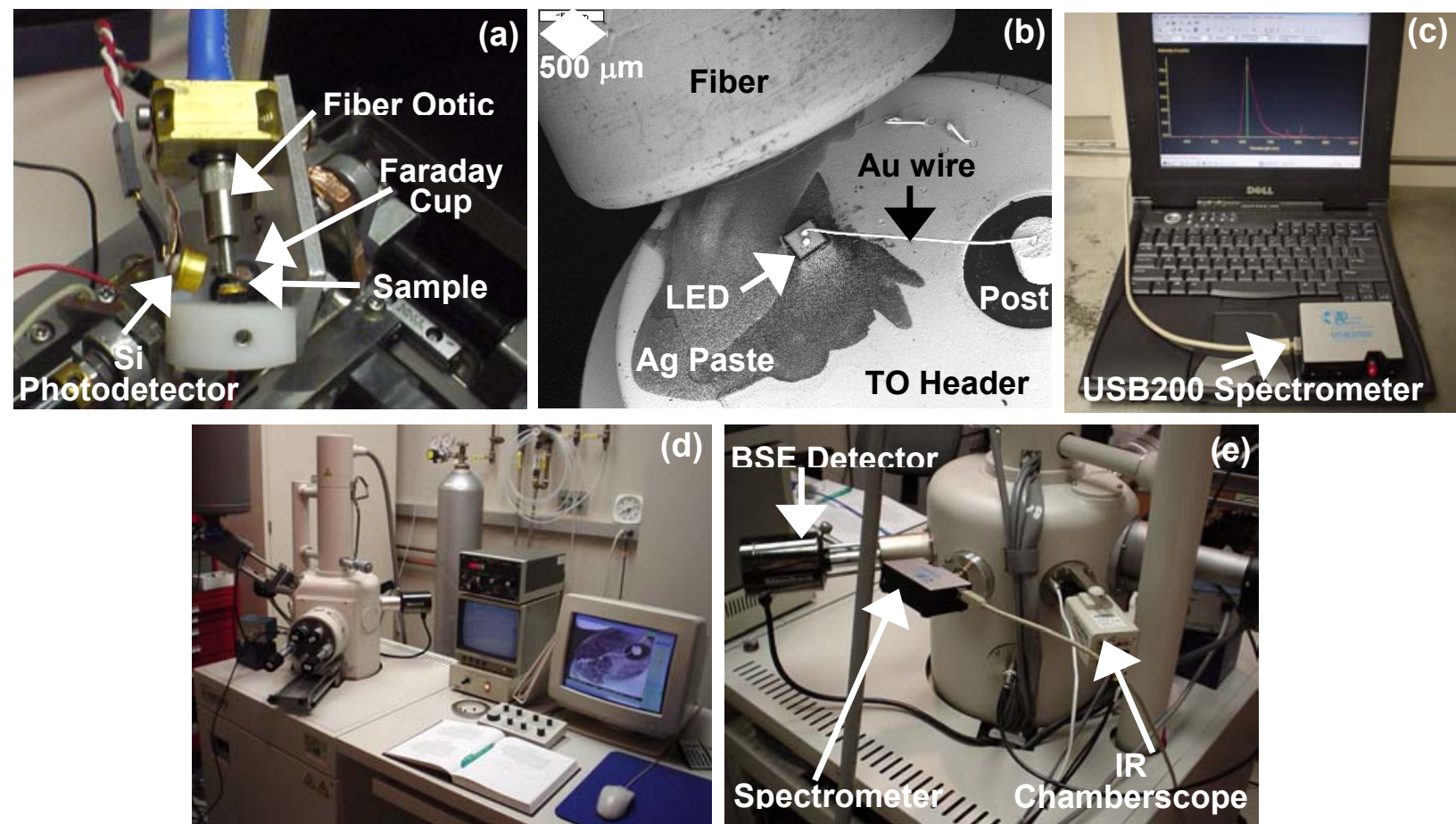

Fig. 1 (a) SEM-CL spectroscopic and panchromatic sample holder design. (b) Secondary electron image of fiber optic collection system. (c) Ocean Optics USB2000 miniature spectrometer. (d) and (e) Hitachi S$3200 \mathrm{~N}$ SEM and position of spectrometer feedthrough.
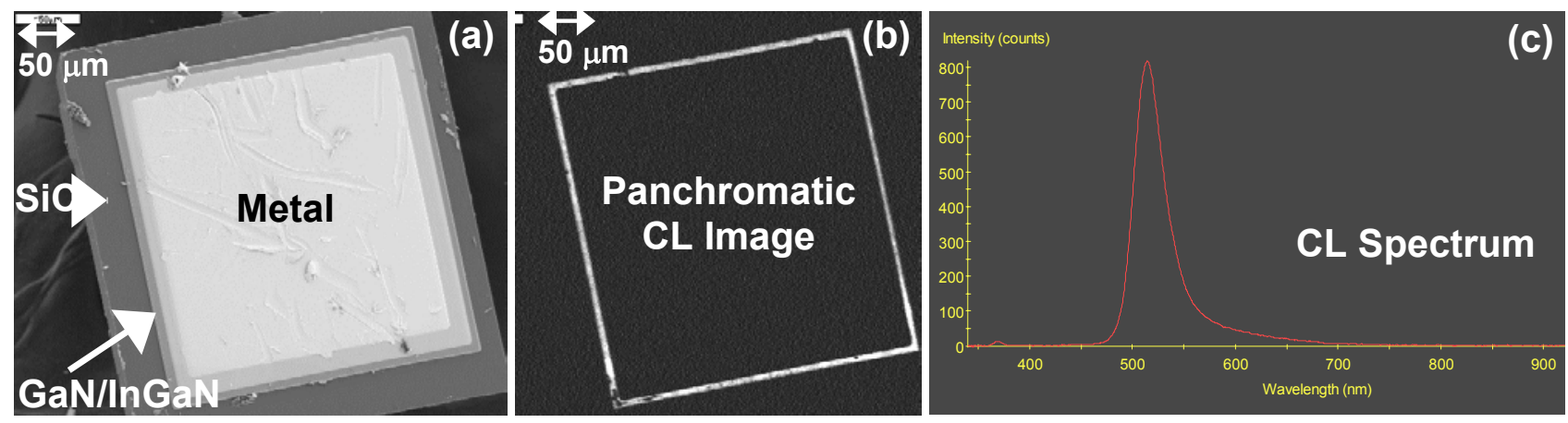

Fig. 2 (a) Secondary electron image and (b) panchromatic CL image of the InGaN-based LED collected at $10 \mathrm{keV}$. (c) CL spectrum displayed with Ocean Optics software.
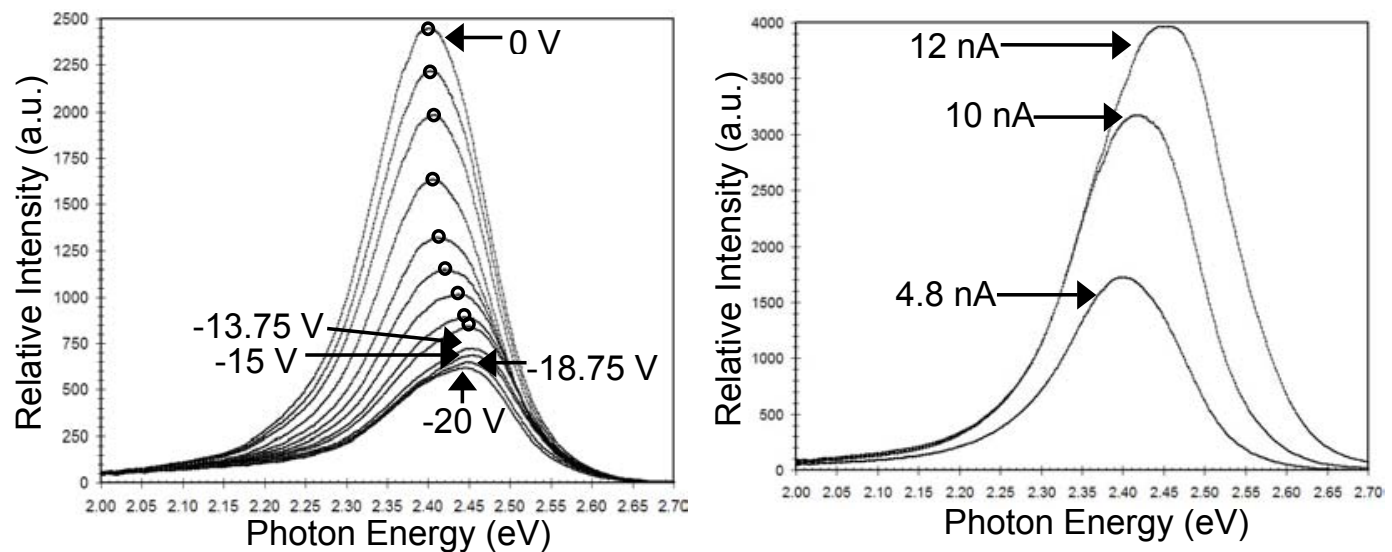

Fig. 3 Room temperature SEM-CL spectra of the InGaN MQW LED collected at (a) several reverse biases showing and (b) at various carrier generation densities or beam currents. 